\title{
Some Inequalities for the Derivative of Polynomials
}

\author{
Sunil Hans, ${ }^{1}$ Dinesh Tripathi, ${ }^{2,3}$ and Babita Tyagi ${ }^{4}$ \\ ${ }^{1}$ Department of Applied Sciences, School of Engineering and Technology, ITM University, Gurgaon 122017, India \\ ${ }^{2}$ Department of Mathematics, Manav Rachna College of Engineering, Faridabad 121004, India \\ ${ }^{3}$ Department of Mathematics and Statistics, Banasthali University, Banasthali Niwai, Rajasthan 304022, India \\ ${ }^{4}$ Department of Mathematics, School of Basic and Applied Sciences, Galgotias University, Greater Noida 201306, India
}

Correspondence should be addressed to Sunil Hans; sunil.hans82@yahoo.com

Received 1 May 2014; Revised 30 August 2014; Accepted 3 September 2014; Published 14 September 2014

Academic Editor: Mohsen Tadi

Copyright (C) 2014 Sunil Hans et al. This is an open access article distributed under the Creative Commons Attribution License, which permits unrestricted use, distribution, and reproduction in any medium, provided the original work is properly cited.

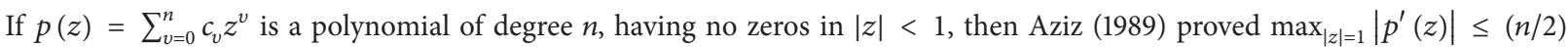
$\left(M_{\alpha}^{2}+M_{\alpha+\pi}^{2}\right)^{1 / 2}$, where $M_{\alpha}=\max _{1 \leq k \leq n}\left|p\left(e^{i(\alpha+2 k \pi) / n}\right)\right|$. In this paper, we consider a class of polynomial $P_{n}^{\mu}$ of degree $n$, defined as $p(z)=a_{0}+\sum_{v=\mu}^{n} a_{v} z^{v}$ and present certain generalizations of above inequality and some other well-known results.

\section{Introduction and Statement of the Results}

Let $P_{n}$ denote the space of all complex polynomials of degree $n$. If $p \in P_{n}$, that is, $p(z)=\sum_{\nu=0}^{n} a_{\nu} z^{v}$, then according to the famous result of Bernstein (see [1])

$$
\max _{|z|=1}\left|p^{\prime}(z)\right| \leq n \max _{|z|=1}|p(z)|
$$

and a simple deduction from the maximum modulus principle (see [2, page 346])

$$
\max _{|z|=1}|p(R z)| \leq R^{n} \max _{|z|=1}|p(z)| .
$$

Both inequalities (1) and (2) are sharp and the equality holds if and only if $p(z)$ has all its zeros at the origin.

If we restrict ourselves to the class of polynomial having no zeros in $|z|<1$, then (1) can be sharpened. In fact, P. Erdös conjectured and later Lax [3] proved that if $p(z) \neq 0$ in $|z|<$ 1 , then

$$
\max _{|z|=1}\left|p^{\prime}(z)\right| \leq \frac{n}{2} \max _{|z|=1}|p(z)|
$$

It was shown by Frappier et al. [4, Theorem 8] that if $p \in P_{n}$, then

$$
\max _{|z|=1}\left|p^{\prime}(z)\right| \leq n \max _{1 \leq \rho \leq 2 n}\left|p\left(e^{i \rho \pi / n}\right)\right|
$$

and clearly (4) represents a refinement of (1), since the maximum of $|p(z)|$ on $|z|=1$ may be larger than maximum of $|p(z)|$ taken over $(2 n)$ th roots of unity as one can show by taking a simple example $p(z)=z^{n}+i a, a>0$.

In this connection, Aziz [5] improved inequality (4) by showing that if $p \in P_{n}$, then for every real $\alpha$

$$
\max _{|z|=1}\left|p^{\prime}(z)\right| \leq \frac{n}{2}\left(M_{\alpha}+M_{\alpha+\pi}\right)
$$

and inequality (2) for $R>1$

$$
\max _{|z|=1}|p(R z)-p(z)| \leq \frac{R^{n}-1}{2}\left(M_{\alpha}+M_{\alpha+\pi}\right),
$$

where

$$
M_{\alpha}=\max _{1 \leq \rho \leq n}\left|p\left(e^{i(\alpha+2 \rho \pi) / n}\right)\right| .
$$

Inequality (3) has also been improved by Aziz [5] by using the same argument as in inequality (5) and he proved that if $p \in P_{n}$ and $p(z) \neq 0$ in $|z|<1$, then for every real $\alpha$

$$
\max _{|z|=1}\left|p^{\prime}(z)\right| \leq \frac{n}{2}\left(M_{\alpha}^{2}+M_{\alpha+\pi}^{2}\right)^{1 / 2}
$$

and also improved inequality (6) for every real $\alpha$ and $R>1$

$$
\max _{|z|=1}|p(R z)-p(z)| \leq \frac{R^{n}-1}{2}\left(M_{\alpha}^{2}+M_{\alpha+\pi}^{2}\right)^{1 / 2},
$$

where $M_{\alpha}$ is same as defined in (7). 
Recently, Rather and Shah [6] considered the class of polynomials having no zeros in $|z|<k, k \geq 1$ and $m=$ $\min _{|z|=1}|p(z)|$ and improved the inequalities (8) and (9) of Aziz [5] by showing the following.

Theorem A. If $p \in P_{n}, p(z) \neq 0$ in $|z|<k, k \geq 1$, and $m=\min _{|z|=k}|p(z)|$, then for every real $\alpha$

$$
\max _{|z|=1}\left|p^{\prime}(z)\right| \leq \frac{n}{\sqrt{2\left(1+k^{2}\right)}}\left(M_{\alpha}^{2}+M_{\alpha+\pi}^{2}-2 m^{2}\right)^{1 / 2}
$$

where $M_{\alpha}$ is defined in (7).

Theorem B. If $p \in P_{n}, p(z) \neq 0$ in $|z|<k, k \geq 1$, and $m=$ $\min _{|z|=k}|p(z)|$, then for every real $\alpha$ and $R>1$

$$
\max _{|z|=1}|p(R z)-p(z)| \leq \frac{R^{n}-1}{\sqrt{2\left(1+k^{2}\right)}}\left(M_{\alpha}^{2}+M_{\alpha+\pi}^{2}-2 m^{2}\right)^{1 / 2},
$$

where $M_{\alpha}$ is defined in (7).

In this paper, we first present the following result, which is a generalization of inequality (10) on class of polynomial $P_{n}^{\mu}$ of degree $n$ defined as $p(z)=a_{0}+\sum_{\nu=\mu}^{n} a_{\nu} z^{\nu}, 0 \leq \mu \leq n$ and having no zeros in $|z|<k, k \geq 1$.

Theorem 1. If $p \in P_{n}^{\mu}, p(z) \neq 0$ in $|z|<k, k \geq 1$, and $m=$ $\min _{|z|=k}|p(z)|$, then for every real $\alpha$

$$
\max _{|z|=1}\left|p^{\prime}(z)\right| \leq \frac{n}{\sqrt{2\left(1+k^{2 \mu}\right)}}\left(M_{\alpha}^{2}+M_{\alpha+\pi}^{2}-2 m^{2}\right)^{1 / 2},
$$

where $M_{\alpha}$ is defined in (7).

On taking $k=1$ in Theorem 1 , the following result has been obtained, which was shown by Rather and Shah [6].

Corollary 2. If $p \in P_{n}, p(z) \neq 0$ in $|z|<1$ and $m=$ $\min _{|z|=1}|p(z)|$, then for every real $\alpha$

$$
\max _{|z|=1}\left|p^{\prime}(z)\right| \leq \frac{n}{2}\left(M_{\alpha}^{2}+M_{\alpha+\pi}^{2}-2 m^{2}\right)^{1 / 2}
$$

where $M_{\alpha}$ is defined in (7).

If we consider that some zeros of the polynomial $p \in P_{n}^{\mu}$ are on $|z|=k$, then $m=0$ and the following result has been obtained from Theorem 1 .

Corollary 3. If $p \in P_{n}^{\mu}$ and $p(z) \neq 0$ in $|z| \leq k, k \geq 1$, then for every real $\alpha$

$$
\max _{|z|=1}\left|p^{\prime}(z)\right| \leq \frac{n}{\sqrt{2\left(1+k^{2 \mu}\right)}}\left(M_{\alpha}^{2}+M_{\alpha+\pi}^{2}\right)^{1 / 2},
$$

where $M_{\alpha}$ is defined in (7).
Remark 4. For $k=1$, Corollary 3 reduces to inequality (8) due to Aziz [5].

Taking $\mu=1$ in inequality (14) of Corollary 3 , we get the following result.

Corollary 5. If $p \in P_{n}$ and $p(z) \neq 0$ in $|z| \leq k, k \geq 1$, then for every real $\alpha$

$$
\max _{|z|=1}\left|p^{\prime}(z)\right| \leq \frac{n}{\sqrt{2\left(1+k^{2}\right)}}\left(M_{\alpha}^{2}+M_{\alpha+\pi}^{2}\right)^{1 / 2}
$$

where $M_{\alpha}$ is defined in (7).

Next result is the generalization of inequality (11) of Rather and Shah [6] for the polynomial $p \in P_{n}^{\mu}$ and having no zeros in $|z|<k, k \geq 1$.

Theorem 6. If $p \in P_{n}^{\mu}, p(z) \neq 0$ in $|z|<k, k \geq 1$, and $m=\min _{|z|=k}|p(z)|$, then for every real $\alpha$ and $R \geq r \geq 1$

$$
\begin{aligned}
& \max _{|z|=1}|p(R z)-p(r z)| \\
& \quad \leq \frac{R^{n}-r^{n}}{\sqrt{2\left(1+k^{2 \mu}\right)}}\left(M_{\alpha}^{2}+M_{\alpha+\pi}^{2}-2 m^{2}\right)^{1 / 2},
\end{aligned}
$$

where $M_{\alpha}$ is defined in (7).

Remark 7. For taking $\mu=1$ and $r=1$, Theorem 6 reduces inequality (11) of Theorem $\mathrm{B}$ and on dividing both sides of inequality (16) by $R-r$ and taking $R \rightarrow r$, inequality (12) of Theorem 1 is obtained.

If we take $k=1$ and $r=1$ in inequality (16), we have the following inequality due to Rather and Shah [6].

Corollary 8. If $p \in P_{n}, p(z) \neq 0$ in $|z|<1$ and $m=$ $\min _{|z|=1}|p(z)|$, then for every real $\alpha$ and $R>1$

$$
\max _{|z|=1}|p(R z)-p(z)| \leq \frac{R^{n}-1}{2}\left(M_{\alpha}^{2}+M_{\alpha+\pi}^{2}-2 m^{2}\right)^{1 / 2}
$$

where $M_{\alpha}$ is defined in (7).

If we take $\mu=1$ in inequality (16) of Theorem 6 , we have the following result.

Corollary 9. If $p \in P_{n}$ and $p(z) \neq 0$ in $|z|<k, k \geq 1$, then for every $\alpha$ and $R \geq r \geq 1$

$\max _{|z|=1}|p(R z)-p(r z)| \leq \frac{R^{n}-r^{n}}{\sqrt{2\left(1+k^{2}\right)}}\left(M_{\alpha}^{2}+M_{\alpha+\pi}^{2}-2 m^{2}\right)^{1 / 2}$,

where $M_{\alpha}$ is defined in (7).

Remark 10. From inequality (18), it is clear that Corollary 9 is a generalization of inequality (11). Also on dividing inequality (18) by $R-r$ and making $R \rightarrow r$ we have inequality (13) of Corollary 2. 
Another generalization of Theorem 6 has been obtained by considering the fact that some zeros of $p \in P_{n}^{\mu}$ are on $|z|=$ $k$; that is, at $m=0$ the following result has been found from Theorem 6 .

Corollary 11. If $p \in P_{n}^{\mu}$ and $p(z) \neq 0$ in $|z| \leq k, k \geq 1$, then for every real $\alpha$ and $R \geq r \geq 1$

$$
\max _{|z|=1}|p(R z)-p(r z)| \leq \frac{R^{n}-r^{n}}{\sqrt{2\left(1+k^{2 \mu}\right)}}\left(M_{\alpha}^{2}+M_{\alpha+\pi}^{2}\right)^{1 / 2},
$$

where $M_{\alpha}$ is defined in (7).

Remark 12. For $k=1$ and $r=1$, Corollary 11 reduces to inequality (9) due to Aziz [5] and dividing inequality (19) by $R-r$ and taking $R \rightarrow r$ we have inequality (14) of Corollary 3 .

An immediate consequence of Corollary 11 has to be obtained by taking $\mu=1$.

Corollary 13. If $p \in P_{n}$ and $p(z) \neq 0$ in $|z| \leq k, k \geq 1$, then for every real $\alpha$ and $R \geq r \geq 1$

$$
\max _{|z|=1}|p(R z)-p(r z)| \leq \frac{R^{n}-r^{n}}{\sqrt{2\left(1+k^{2}\right)}}\left(M_{\alpha}^{2}+M_{\alpha+\pi}^{2}\right)^{1 / 2},
$$

where $M_{\alpha}$ is defined in (7).

The following result also gives a generalization of inequality (19) by taking $k=1$ in Corollary 11.

Corollary 14. If $p \in P_{n}$ and $p(z) \neq 0$ in $|z| \leq k, k \geq 1$, then for every real $\alpha$ and $R \geq r \geq 1$

$$
\max _{|z|=1}|p(R z)-p(r z)| \leq \frac{R^{n}-r^{n}}{2}\left(M_{\alpha}^{2}+M_{\alpha+\pi}^{2}\right)^{1 / 2},
$$

where $M_{\alpha}$ is defined in (7).

If we consider a class of polynomials $p(z)=z^{s}\left(a_{0}+\right.$ $\left.\sum_{\nu=\mu}^{v=n-s} a_{\nu} z^{\nu}\right), 1 \leq \mu \leq n-s, 0 \leq s \leq n-1$, and having $s$-fold zeros at origin and remaining $n-s$ zeros in $|z| \geq k, k \geq 1$, then we prove the following.

Theorem 15. If $p(z)=z^{s}\left(a_{0}+\sum_{\nu=\mu}^{\nu=n-s} a_{\nu} z^{\nu}\right), 1 \leq \mu \leq n-s$, $0 \leq s \leq n-1$ is a polynomial of degree $n$ and having $s$-fold zeros at origin and remaining $n-s$ zeros in $|z| \geq k, k \geq 1$, then for every $\alpha$

$$
\begin{aligned}
\max _{|z|=1}\left|p^{\prime}(z)\right| \leq & s \max _{|z|=1}|p(z)| \\
& +\frac{(n-s)}{\sqrt{2\left(1+k^{2 \mu}\right)}}\left(M_{\alpha}^{* 2}+M_{\alpha+\pi}^{* 2}\right)^{1 / 2}
\end{aligned}
$$

where

$$
M_{\alpha}^{*}=\max _{1 \leq \rho \leq n-s}\left|P\left(e^{i(\alpha+2 \rho \pi) / n-s}\right)\right| .
$$

Remark 16. If there are no zeros at origin, that is if $s=0$, then inequality (22) is reducing to inequality (14) of Corollary 3.

As an application of inequality (22), we obtained the following results by considering $\mu=1$ and $k=1$, respectively, which are generalization of inequality (15) of Corollary 5 and inequality (8) due to Aziz [5] on $s$-fold zeros, respectively.

Corollary 17. If $p \in P_{n}$ and having s-fold zeros at origin and remaining $n-s$ zeros in $|z| \geq k, k \geq 1$, then for every $\alpha$

$$
\max _{|z|=1}\left|p^{\prime}(z)\right| \leq s \max _{|z|=1}|p(z)|+\frac{(n-s)}{\sqrt{2\left(1+k^{2}\right)}}\left(M_{\alpha}^{* 2}+M_{\alpha+\pi}^{* 2}\right)^{1 / 2}
$$

where $M_{\alpha}^{*}$ is defined in (23).

Corollary 18. If $p \in P_{n}$, having s-fold zeros at origin and remaining $n-s$ zeros in $|z| \geq 1$, then for every $\alpha$

$$
\max _{|z|=1}\left|p^{\prime}(z)\right| \leq s \max _{|z|=1}|p(z)|+\frac{n}{2}\left(M_{\alpha}^{* 2}+M_{\alpha+\pi}^{* 2}\right)^{1 / 2}
$$

where $M_{\alpha}^{*}$ is defined in (23).

Inequality (22) of Theorem 15 can be improved by including $m=\min _{|z|=k}|p(z)|$. In this connection, instead of proving Theorem 15, we prove the following more general result.

Theorem 19. If $p(z)=z^{s}\left(a_{0}+\sum_{\nu=\mu}^{\nu=n-s} a_{\nu} z^{\nu}\right), 1 \leq \mu \leq n-s$, $0 \leq s \leq n-1$ is a polynomial of degree $n$ and having $s$-fold zeros at origin and remaining $n-s$ zeros in $|z| \geq k, k \geq 1$, then for every real $\alpha$ and $m=\min _{|z|=k}|p(z)|$

$$
\begin{aligned}
\max _{|z|=1}\left|p^{\prime}(z)\right| \leq & s \max _{|z|=1}|p(z)| \\
& +\frac{(n-s)}{\sqrt{2\left(1+k^{2 \mu}\right)}}\left\{M_{\alpha}^{* 2}+M_{\alpha+\pi}^{* 2}-2 \frac{m^{2}}{k^{2 s}}\right\}^{1 / 2},
\end{aligned}
$$

where $M_{\alpha}^{*}$ is defined in (23).

By taking $\mu=1$ in inequality (26) of Theorem 15, we obtained the following.

Corollary 20. If $p \in P_{n}$, having $s$-fold zeros at origin and remaining $n-s$ zeros in $|z| \geq k, k \geq 1$, then for every real $\alpha$ and $m=\min _{|z|=k}|p(z)|$

$$
\begin{aligned}
\max _{|z|=1}\left|p^{\prime}(z)\right| \leq & s \max _{|z|=1}|p(z)| \\
& +\frac{(n-s)}{\sqrt{2\left(1+k^{2}\right)}}\left\{M_{\alpha}^{* 2}+M_{\alpha+\pi}^{* 2}-2 \frac{m^{2}}{k^{2 s}}\right\}^{1 / 2},
\end{aligned}
$$

where $M_{\alpha}^{*}$ is defined in (22).

And for $k=1$ inequality (26) becomes as follows. 
Corollary 21. If $p \in P_{n}$, having s-fold zeros at origin and remaining $n-s$ zeros in $|z| \geq 1$, then for every real $\alpha$ and $m=\min _{|z|=1}|p(z)|$

$$
\begin{aligned}
\max _{|z|=1}\left|p^{\prime}(z)\right| \leq & s \max _{|z|=1}|p(z)| \\
& +\frac{(n-s)}{2}\left(M_{\alpha}^{* 2}+M_{\alpha+\pi}^{* 2}-2 m^{2}\right)^{1 / 2},
\end{aligned}
$$

where $M_{\alpha}^{*}$ is defined in (22).

Remark 22. Corollaries 20 and 21 are generalization of inequalities (11) and (9) for $s$-fold zeros of $p \in P_{n}$ and for $s=0$, Theorem 19 gives inequality (12) of Theorem 1 .

\section{Lemmas}

For the proof of above results, the following lemmas are required. The very first lemma is due to Aziz [5].

Lemma 23. If $p \in P_{n}$, then for $|z|=1$ and for every real $\alpha$

$$
\left|p^{\prime}(z)\right|^{2}+\left|n p(z)-z p^{\prime}(z)\right|^{2} \leq \frac{n^{2}}{2}\left(M_{\alpha}^{2}+M_{\alpha+\pi}^{2}\right),
$$

where $M_{\alpha}$ is defined the same as in (7).

Next lemma is due to Aziz and Rather [7].

Lemma 24. If $p \in P_{n}^{\mu},\left|p^{\prime}(z)\right| \neq 0$ in $|z|<k, k \geq 1$, and $q(z)=z^{n} \overline{p(1 / \bar{z})}$, then for $|z|=1$ and $R>1$

$$
k^{\mu}|p(R z)-p(z)| \leq|q(R z)-q(z)|-\left(R^{n}-1\right) m,
$$

where $m=\min _{|z|=k}|p(z)|$.

\section{Proof of the Theorems}

Proof of Theorem 1. By the hypothesis $p(z) \neq 0$ in $|z|<k$, $k \geq 1$, dividing (30) by $R-1$ and taking $R \rightarrow 1$ in Lemma 24 we have, for $|z|=1$,

$$
\left(k^{\mu}\left|p^{\prime}(z)\right|+n m\right)^{2} \leq\left|q^{\prime}(z)\right|^{2} .
$$

Since

$$
\begin{aligned}
\left(k^{\mu}\left|p^{\prime}(z)\right|+n m\right)^{2} & =k^{2 \mu}\left|p^{\prime}(z)\right|^{2}+n^{2} m^{2}+2 n m k^{2 \mu}|p(z)| \\
& \geq k^{2 \mu}\left|p^{\prime}(z)\right|^{2}+n^{2} m^{2}
\end{aligned}
$$

and for $|z|=1,\left|q^{\prime}(z)\right|=\left|n p(z)-z p^{\prime}(z)\right|$; therefore inequality (31) gives

$$
k^{2 \mu}\left|p^{\prime}(z)\right|^{2}+n^{2} m^{2} \leq\left|n p(z)-z p^{\prime}(z)\right|^{2}
$$

and using Lemma 23 in inequality (33), we have

$$
\left(1+k^{2 \mu}\right)\left|p^{\prime}(z)\right|^{2}+n^{2} m^{2} \leq \frac{n^{2}}{2}\left(M_{\alpha}^{2}+M_{\alpha+\pi}^{2}\right)
$$

which completes the proof of Theorem 1 .
Proof of Theorem 6. Applying the result (2) to the polynomial $p^{\prime}(z)$, which is of degree $n-1$, and using (12), we obtained for $t \geq 1$ and $0 \leq \theta<2 \pi$

$$
\begin{aligned}
\left|p^{\prime}\left(t e^{i \theta}\right)\right| & \leq t^{n-1} \max _{|z|=1}\left|p^{\prime}(z)\right| \\
& \leq t^{n-1} \frac{n}{\sqrt{2\left(1+k^{2 \mu}\right)}}\left(M_{\alpha}^{2}+M_{\alpha+\pi}^{2}-2 m^{2}\right)^{1 / 2} .
\end{aligned}
$$

Hence for each $\theta, 0 \leq \theta<2 \pi$ and $R \geq r \geq 1$, we have

$$
\begin{aligned}
\left|p\left(R e^{i \theta}\right)-p\left(r e^{i \theta}\right)\right| & =\left|\int_{r}^{R} e^{i \theta} p^{\prime}\left(t e^{i \theta}\right) d t\right| \\
& \leq \int_{r}^{R}\left|p^{\prime}\left(t e^{i \theta}\right)\right| d t
\end{aligned}
$$

and, from inequality (35), we have

$$
\begin{aligned}
& \left|p\left(R e^{i \theta}\right)-p\left(r e^{i \theta}\right)\right| \\
& \leq \frac{1}{\sqrt{2\left(1+k^{2 \mu}\right)}}\left(M_{\alpha}^{2}+M_{\alpha+\pi}^{2}-2 m^{2}\right)^{1 / 2} \int_{r}^{R} n t^{n-1} \\
& \quad \leq \frac{R^{n}-r^{n}}{\sqrt{2\left(1+k^{2 \mu}\right)}}\left(M_{\alpha}^{2}+M_{\alpha+\pi}^{2}-2 m^{2}\right)^{1 / 2}
\end{aligned}
$$

which implies that, for $|z|=1$ and $R \geq r \geq 1$,

$$
|p(R z)-p(r z)| \leq \frac{R^{n}-r^{n}}{\sqrt{2\left(1+k^{2 \mu}\right)}}\left(M_{\alpha}^{2}+M_{\alpha+\pi}^{2}-2 m^{2}\right)^{1 / 2} \text {. }
$$

Theorem 6 is completed.

Proof of Theorem 19. Let

$$
p(z)=z^{s} \phi(z)
$$

where $\phi(z)=a_{0}+\sum_{\nu=\mu}^{n-s} a_{\nu} z^{\nu}, 1 \leq \mu \leq n-s$, is a polynomial of degree $n-s$, having no zero in $|z|<k, k \geq 1$.

From (39) we have

$$
\begin{aligned}
z p^{\prime}(z) & =s z^{s} \phi(z)+z^{s+1} \phi^{\prime}(z) \\
& =s p(z)+z^{s+1} \phi^{\prime}(z)
\end{aligned}
$$

and for $|z|=1$,

$$
\left|p^{\prime}(z)\right| \leq s|p(z)|+\left|\phi^{\prime}(z)\right|
$$

and the above inequality holds for all point on $|z|=1$.

Since $\phi(z)$ is polynomial of degree $n-s$ and from (39) it can easily be obtained that for $|z|=1,|p(z)|=|\phi(z)|$ and

$$
\min _{|z|=k}|\phi(z)|=\frac{1}{k^{s}} \min _{|z|=k}|p(z)|=\frac{m}{k^{s}}
$$


and then from Theorem 15 (inequality (18)), we have

$$
\max _{|z|=1}\left|\phi^{\prime}(z)\right| \leq \frac{n-s}{\sqrt{2\left(1+k^{2 \mu}\right)}}\left\{M_{\alpha}^{*^{2}}+M_{\alpha+\pi}^{*^{2}}-\frac{2 m^{2}}{k^{2 s}}\right\}^{1 / 2}
$$

on combining inequality (41) and (43); the proof of Theorem 19 is completed.

\section{Conflict of Interests}

The authors declare that there is no conflict of interests regarding the publication of this paper.

\section{References}

[1] A. C. Schaeffer, "Inequalities of A. Markoff and S. Bernstein for polynomials and related functions," Bulletin of the American Mathematical Society, vol. 47, pp. 565-579, 1941.

[2] M. Riesz, “Über Einen Sat'z des Herrn Serge Bernstein,” Acta Mathematica, vol. 40, pp. 337-347, 1916.

[3] P. D. Lax, "Proof of a conjecture of P. Erdös on the derivative of a polynomial," Bulletin of the American Mathematical Society, vol. 50, pp. 509-513, 1944.

[4] C. Frappier, Q. I. Rahman, and S. Ruscheweyh, "New inequalities for polynomials," Transactions of the American Mathematical Society, vol. 288, no. 1, pp. 69-99, 1985.

[5] A. Aziz, "A refinement of an inequality of S. Bernstein," Journal of Mathematical Analysis and Applications, vol. 144, no. 1, pp. 226-235, 1989.

[6] N. A. Rather and M. A. Shah, "On the derivative of a polynomial," Applied Mathematics, vol. 3, pp. 746-749, 2012.

[7] A. Aziz and N. A. Rather, "New $L_{q}$ inequalities for polynomials," Mathematical Inequalities \& Applications, vol. 1, no. 2, pp. 177191, 1998. 


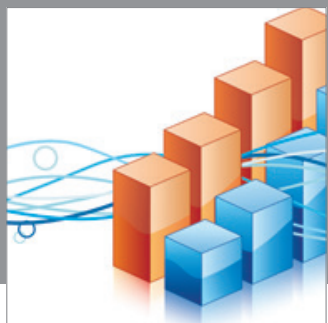

Advances in

Operations Research

mansans

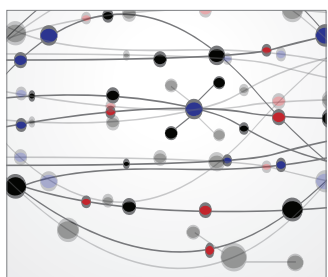

The Scientific World Journal
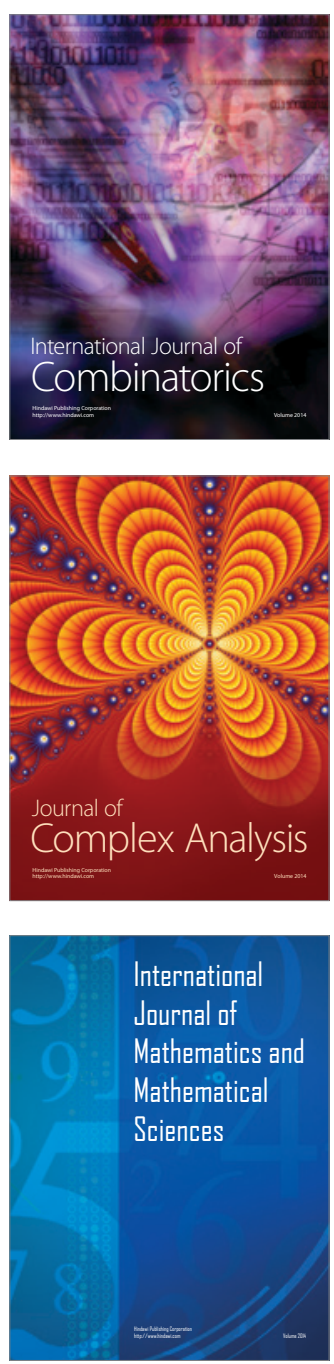
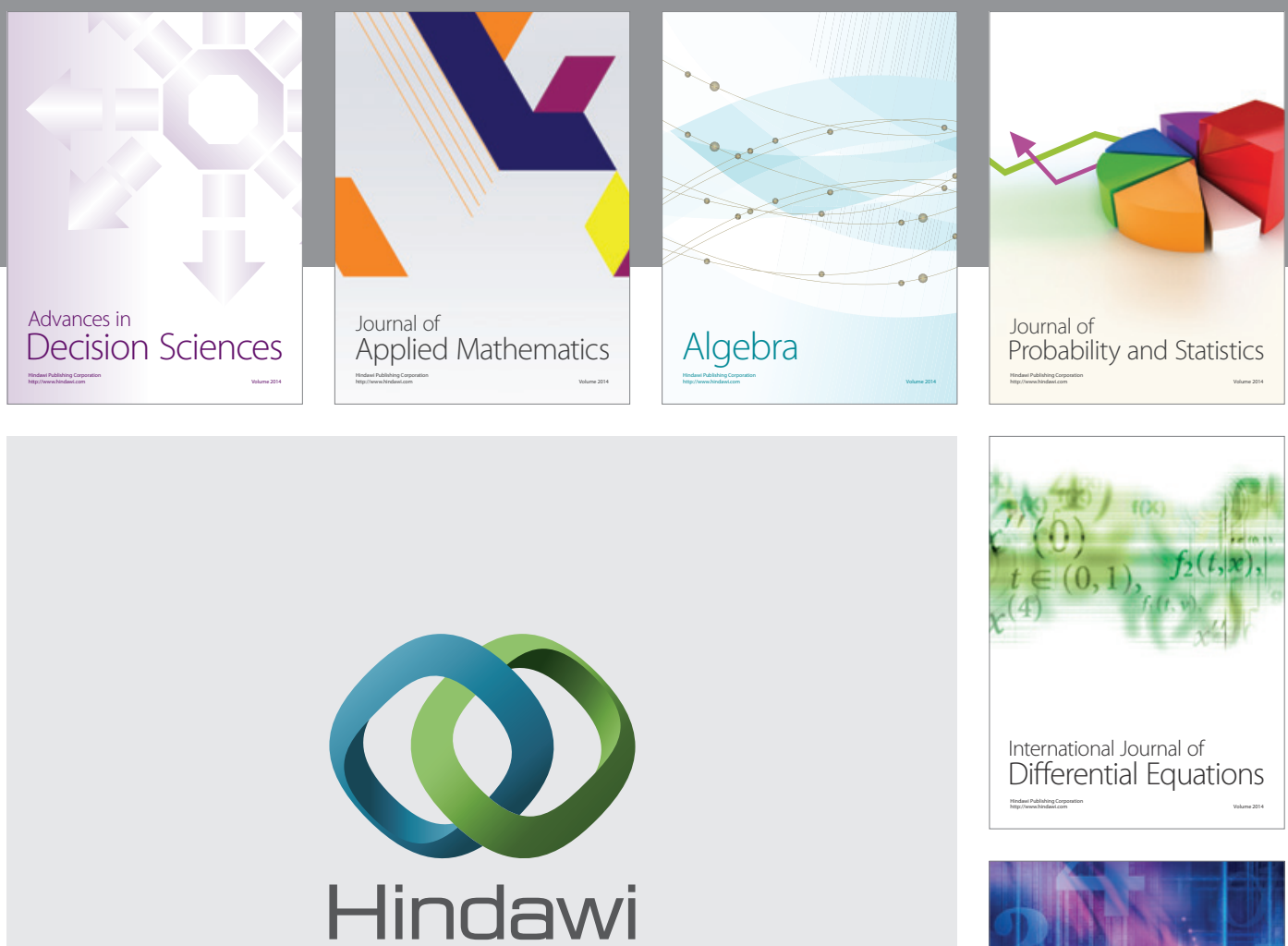

Submit your manuscripts at http://www.hindawi.com
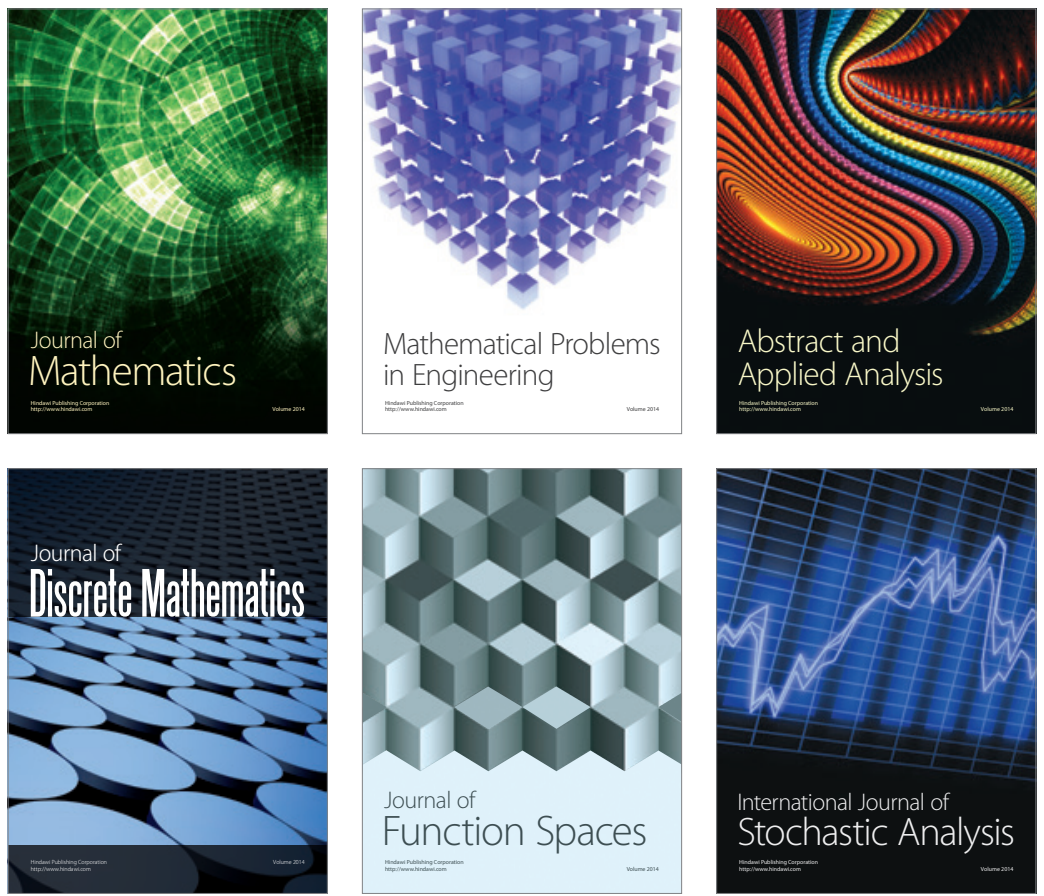

Journal of

Function Spaces

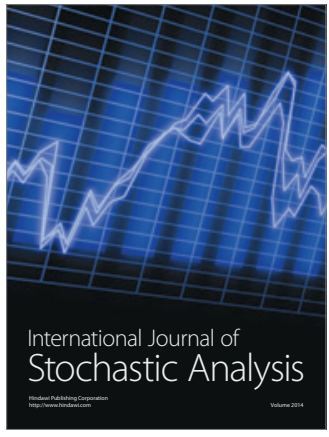

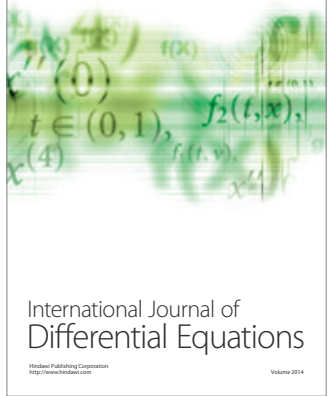
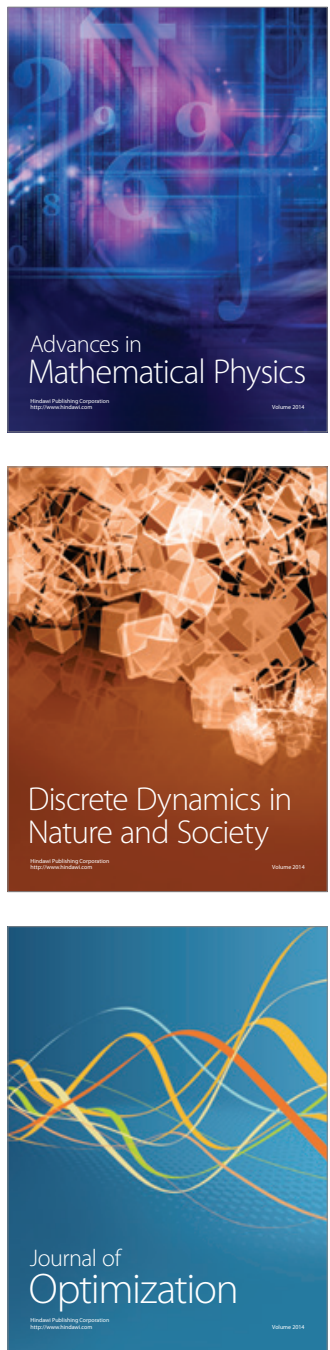